gynäkologische Untersuchung inkl. äußeres Genitale, Scheide und Zervix mit Kolposkopie, Zytologie und - falls notwendig - Biopsie. Eine Beinschwellung und Seitendifferenz wird evaluiert bzw. ausgeschlossen. Bei der rektovaginalen bimanuellen Untersuchung werden vor allem das Parametrium und gesamte kleine Becken ausgetastet. Tumormarker machen nur Sinn, wenn sie primär erhöht sind (SCC beim Plattenepithelkarzinom oder CA 125 und CEA beim Adenokarzinom).

Eine Ultraschalluntersuchung der Nieren sollte erfolgen. Zusätzliche Maßnahmen wie Bildgebung, Zystoskopie, Rektoskopie oder Laparoskopie sind nur bei Verdacht auf ein Rezidiv angezeigt. Rund $80 \%$ der Rezidive fallen durch Symptome auf und nur $25 \%$ werden im Rahmen der standardisierten Nachsorge erfasst.

Die Nachsorge nach Radiochemotherapie sollte in den ersten 2 Jahren 4-mal jährlich, dann halbjährlich stattfinden. Neben der Erfassung möglicher erkrankungsbedingter Symptomatik sollten mögliche Spätnebenwirkungen erfragt, dokumentiert und wenn möglich behandelt werden. Diese können das Lymphsystem (Lymphödeme), Funktionsstörungen der Harnblase und Veränderungen des Stuhlverhaltens sowie hormonelle Entzugssymptomatiken durch die radiogene Schädigung der Ovarien beinhalten.

\section{"Die Nachsorge nach Radio- chemotherapie sollte in den ersten 2 Jahren 4-mal jährlich, dann halbjährlich stattfinden "}

Der routinemäßige Einsatz der Abrasio nach Radiochemotherapie wird kontrovers diskutiert. Aufgrund der Limitationen der bildgebenden Verfahren zur Bestimmung des Remissionsstatus kann die Abrasio hilfreich sein, um Patientinnen, die ggf. bei Tumorpersistenz von einer sekundären Hysterektomie profitieren, selektionieren zu können.

Die rasante technologische Entwicklung in der Radioonkologie hat dazu ge-

\title{
Die Geschichte der HeLa-Zellen
}

Es ist Februar 1951. In der Frauenklinik von Baltimore liegt eine 31-jährige Frau mit Zervixkarzinom auf dem Operationstisch.

Der diensthabende Chirurg, bereitet sich auf eine Radiumtherapie vor. Doch als erstes schneidet er der Patientin mit einem Skalpell zwei münzgroße Gewebestücke vom Gebärmutterhals - eines aus dem Tumor, ein anderes aus dem benachbarten gesunden Gewebe heraus. Die Gewebeproben lässt er einem Zellforscher zukommen. Es sind die

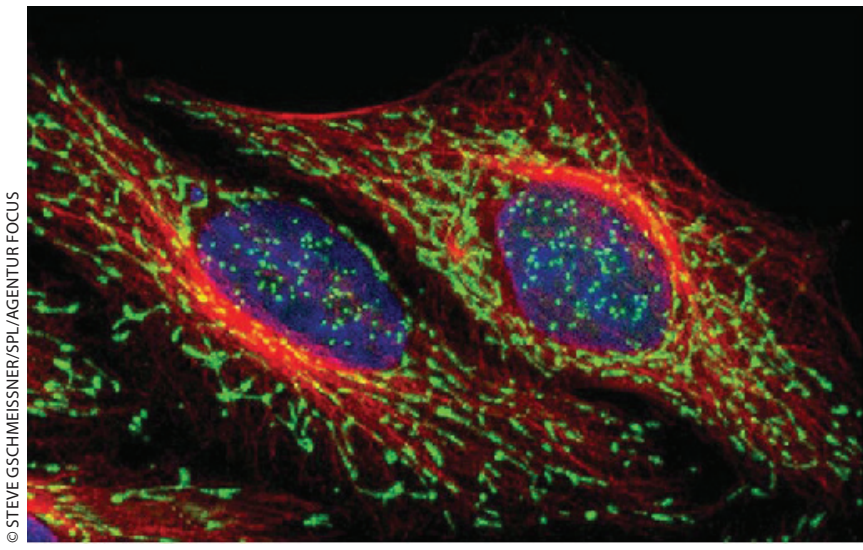

4 Konfokale Mikroskopie von $\mathrm{He}$ La-Zellen: Mitochondrien (grün), a-Tubulin (rot) und Zellkern (blau); aus: Bottone MG et al. (2007) Histochem Cell Biol 127:263271

ersten humanen Zellen, aus denen eine permanente Zelllinie entsteht. Bis zu jenen Wochen war es noch niemandem gelungen, menschliche Zellen künstlich am Leben zu erhalten. Die Krebszellen von Henrietta Lacks (Abkürzung HeLa-Zellen) begannen sich jedoch in Geys Labor nach wenigen Tagen zu teilen. Die Zahl der Zellen verdoppelte sich alle 24 Stunden. In weltweit Dutzenden Laboren vermehrten sich die Zellen weiter. Heute übertrifft ihre Gesamtmasse die Körpermasse der unfreiwilligen Spenderin um mehr als das Hundertfache. Im Laufe der Jahrzehnte bildeten die HeLa-Zellen die Grundlage für wichtige medizinische Forschungen. Unter ihrer Verwendung wurden der Polioimpfstoff sowie Arzneien gegen Parkinson und Leukämie entwickelt.

Henrietta Lacks starb noch 1951 an den Folgen ihrer Krebserkrankung. Weder sie noch ihr Mann, noch ihre fünf Kinder und Enkelkinder erhielten auch nur einen Cent aus den Gewinnen, die mit HeLa-Zellen verdient wurden. Nun wurde ihr mit einer in elfjähriger Arbeit entstandenen Biographie und Medizingeschichte ein Denkmal gesetzt.

$>$ Rebecca Skloot

\section{Die Unsterblickeit der Henrietta Lacks}

Irisiana Verlag. München 2010. 512 Seiten. 19,90 Euro.

führt, dass mittels neuer Techniken wie der intensitätsmodulierten Radiotherapie (IMRT), der Tomotherapie u. a. sowohl die Häufigkeit wie Schwere von radiogenen Akut- und Spätreaktionen gesenkt werden konnten und auf der anderen Seite die Chance für eine Dosiseskalation und Verbesserung der lokalen Kontrolle besteht.

\section{Fazit für die Praxis}

- Die Behandlung von Frauen mit histologisch gesichertem Gebärmutterhalskrebs erfolgt krankheitsbasiert und individuell auf die Erkrankte zugeschnitten.

- Das laparoskopische Staging zur Evaluierung des Lymphknotenstatus stellt den wichtigsten Schritt für die Therapieentscheidung dar. 